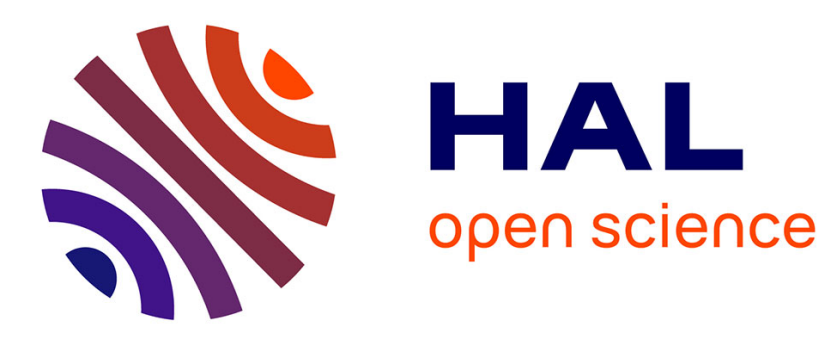

\title{
Kernel Metrics on Normal Cycles and Application to Curve Matching
}

Pierre Roussillon, Joan Alexis Glaunès

\section{To cite this version:}

Pierre Roussillon, Joan Alexis Glaunès. Kernel Metrics on Normal Cycles and Application to Curve Matching. MFCA 2015: 5th MICCAI workshop on Mathematical Foundations of Computational Anatomy, Oct 2015, Munich, Germany. hal-01221101v2

\section{HAL Id: hal-01221101 \\ https://hal.science/hal-01221101v2}

Submitted on 14 Dec 2016

HAL is a multi-disciplinary open access archive for the deposit and dissemination of scientific research documents, whether they are published or not. The documents may come from teaching and research institutions in France or abroad, or from public or private research centers.
L'archive ouverte pluridisciplinaire HAL, est destinée au dépôt et à la diffusion de documents scientifiques de niveau recherche, publiés ou non, émanant des établissements d'enseignement et de recherche français ou étrangers, des laboratoires publics ou privés. 


\title{
Kernel Metrics on Normal Cycles and Application to Curve Matching
}

\author{
Pierre Roussillon and Joan Alexis Glaunès \\ MAP5, UMR 8145 CNRS, Université Paris Descartes, Sorbonne Paris Cité, France \\ pierre.roussillon@parisdescartes.fr alexis.glaunes@parisdescartes.fr
}

\begin{abstract}
In this work we introduce a new dissimilarity measure for shape registration using the notion of normal cycles, a concept from geometric measure theory which allows to generalize curvature for non smooth subsets of the euclidean space. Our construction is based on the definition of kernel metrics on the space of normal cycles which take explicit expressions in a discrete setting. This approach is closely similar to previous works based on currents and varifolds [13|5]. We derive the computational setting for discrete curves in $\mathbb{R}^{3}$, using the Large Deformation Diffeomorphic Metric Mapping framework as model for deformations. We present synthetic experiments and compare with the currents and varifolds approaches.
\end{abstract}

\section{Introduction}

Many applications in medical image analysis require a coherent alignment of images as a pre-processing step, using efficient rigid or non-rigid registration algorithms. Moreover, in the field of computational anatomy, the estimation of optimal deformations between images, or geometric structures segmented from the images, is a building block for any statistical analysis of the anatomical variability of organs. Non-rigid registration is classically tackled down by minimizing a functional composed of two terms, one enforcing regularity of the mapping, and the data-attachment term which evaluates dissimilarity between shapes. Defining good data-attachment terms is important, as it may improve the minimization process, and focus the registration on the important features of the shapes to be matched.

In [13]9 a new framework for dissimilarity measures between sub-manifolds was proposed using kernel metrics defined on spaces of currents. This setting is now commonly used in computational anatomy ; its advantages lie in its simple implementation and the fact that it provides a common framework for continuous and discrete shapes (see [7] for a computational analysis of currents and their numerical implementation). However, currents are oriented objects and thus a consistent orientation of shapes is needed for a coherent matching. Moreover, due to this orientation property, artificial cancellation can occur with shapes with high local variations. To deal with this problem, a more advanced model based on varifolds has been introduced recently [4]. Varifolds are measures over fields of non-oriented linear subspaces. See 4, chap. 3 for an exhaustive analysis. 
In this work, we propose to use a second-order model called normal cycle for defining shape dissimilarities. The normal cycle of a submanifold $X$ is the current associated with its normal bundle $\mathcal{N}_{X}$. The normal cycle encodes second order, i.e. curvature information of $X$; more precisely one can compute integrals of curvatures by evaluating the normal cycle over simple differential forms. Moreover, it has a canonical orientation which is independent of the orientation of $X$ (in fact $X$ does not need to be oriented).

Our approach is closely related to the currents and varifolds models in that it is based on the definition of kernel metrics that take explicit form in a discrete setting. This paper is organized as follows : in Sect. 1 and 2 we introduce the mathematical notions of currents and normal cycles and define the kernel metric in a general setting. In Sect. 3 we derive explicit formulas for the metric in the case of discrete curves in $\mathbb{R}^{3}$. In Sect. 4 we introduce the general curve matching problem and recall some basic facts about the diffeomorphic model. Finally we present two sets of synthetic experiments in Sect. 5

\section{Currents and Normal Cycles}

\subsection{Currents}

We recall here the definition of current used in [7], definition 1.2.

\section{Definition 1 (Currents).}

The space of $m$-currents in $\mathbb{R}^{d}$ is defined as the topological dual $\Omega_{0}^{m}\left(\mathbb{R}^{d}\right)^{\prime}$, where $\Omega_{0}^{m}\left(\mathbb{R}^{d}\right):=\mathcal{C}_{0}^{0}\left(\mathbb{R}^{d},\left(\Lambda^{m} \mathbb{R}^{d}\right)^{*}\right)$ is the space of continuous $m$-differential forms vanishing at infinity, with the supremum norm : $\|\omega\|_{\infty}=\sup _{x \in \mathbb{R}^{d}}\left|\omega_{x}\right|$. A $m$-current is thus a linear map $T: \Omega_{0}^{m}\left(\mathbb{R}^{d}\right) \rightarrow \mathbb{R}$ such that there exists $C_{T}>0$ such that for every differential form $\omega$,

$$
T(\omega) \leq C_{T}\|\omega\|_{\infty}
$$

Example 1. A fundamental example of current (which will be useful when dealing with discrete shape) is the "Dirac" current. Let $x \in \mathbb{R}^{d}, \alpha \in \Lambda^{m}\left(\mathbb{R}^{d}\right)$. For $\omega \in \Omega_{0}^{m}\left(\mathbb{R}^{d}\right)$, we define $\delta_{x}^{\alpha}(\omega):=\omega_{x}(\alpha)$.

Any sufficiently regular shape in $\mathbb{R}^{d}$ can be seen as a current. Let $\mathcal{H}^{m}$ be the $m$-dimensional Hausdorff measure in $\mathbb{R}^{d}$. If $X$ is a regular $m$-dimensional submanifold, $\mathcal{H}^{m}$ coincides on $X$ with the volume form of $X$. Assume $X$ is a compact, oriented, $m$-rectifiable set (see definition in [8]). Then the tangent space $T_{x} X$ exists for $\mathcal{H}^{m}$-almost every $x \in X$, and one can associate to $X$ a current $[X]$, defined as :

$$
[X](\omega):=\int_{X} \omega_{x}\left(\tau_{X}(x)\right) d \mathcal{H}^{m}(x)
$$

where $\tau_{X}(x)=\tau_{1}(x) \wedge \cdots \wedge \tau_{m}(x)$, with $\left(\tau_{i}(x)\right)_{1 \leq i \leq m}$ an orthonormal basis of $T_{x} X$. If we consider the opposite orientation of $X: \tilde{X}$, we have $[\tilde{X}]=-[X]$. 


\subsection{Normal Cycles}

Normal cycles find their roots in the seminal work of Federer. In [8], he proved that for a set with positive reach (see definition below), the volume of the $\varepsilon$ parallel neighbour of $X \cap B$ (where $B$ is a borelian) can be expressed as a polynomial of $\varepsilon$; and more importantly that the coefficients of this polynomial can be interpreted as curvature measures of the set $X$. These measures have integral representation, and Zähle in [15] introduced $d-1$ generalized principal curvatures for sets with positive reach, and retrieved Federer's curvature measures by integrating functions of these principal curvatures over the unit normal bundle. She showed that this can be done by integrating adequate differential forms on the associated current : this is exactly the normal cycle. This work was pushed forward in [16. The book of Morvan (12]) is a self-sufficient reference for normal cycles as they will be used in this paper. Note that normal cycles have already been applied for computational analysis of discrete surfaces in [6]. Cohen-Steiner and Morvan derive a definition of discrete curvature and discrete curvature tensor for polyhedral surfaces based on the normal cycle.

We follow Federer $[8$ to give a definition of the normal bundle and the normal cycle in the context of sets with positive reach.

Definition 2 (Sets with positive reach). Let $X \subset \mathbb{R}^{d}$ and for any $\varepsilon>0$, denote $X_{\varepsilon}=\left\{x \in \mathbb{R}^{d}, d(x, X) \leq \varepsilon\right\}$ the $\varepsilon$ tube around $X$. The reach of $X$ is the supremum of $r>0$ such that for every $0<\varepsilon \leq r$, there exists a unique projection of $x \in X_{\varepsilon}$ onto $X . X$ is said to be a positive reach set if $r>0$.

On a set with positive reach $R$, one can roll a ball of radius less than $R$. Thus, a set with positive reach can be seen heuristically as a set with a bounded below curvature.

Definition 3 (Unit Normal bundle). Let $X$ be a set with positive reach. We define the tangent cone, the unit normal cone at $x \in X$ and the unit normal bundle respectively as

$$
\begin{aligned}
& \operatorname{Tan}(X, x)=\left\{v \in \mathbb{R}^{d}|\forall \varepsilon>0| \exists y \in B(x, \varepsilon) \cap X,|c(y-x)-v|<\varepsilon\right\}, \\
& \operatorname{Nor}(X, x)=\left\{u \in \mathbb{R}^{d} \mid \forall v \in \operatorname{Tan}(X, x),\langle u, v\rangle \leq 0\right\} \cap S^{d-1}, \\
& \mathcal{N}_{X}=\{(x, n), x \in X, n \in \operatorname{Nor}(X, x)\} .
\end{aligned}
$$

For a $\mathcal{C}^{2}$-submanifold, the unit normal bundle defined here coincides with the classical one, which is a $(d-1)$-submanifold in the $(2 d-1)$ dimensional manifold $\mathbb{R}^{d} \times S^{d-1}$. More generally, $\mathcal{N}_{X}$ is a $(d-1)$-rectifiable set in $\mathbb{R}^{d} \times S^{d-1}$ when $X$ has positive reach and $\partial X_{\varepsilon}$ is a $(d-1)$-dimensional differentiable submanifold (hypersurface), with Lipschitzian unit normal vector field (see again [8], 4.8).

Example 2 (Unit normal bundle of a curve in $\mathbb{R}^{3}$ ). Let $\gamma:[0, L] \rightarrow \mathbb{R}^{3}$ be a parametrized curve in $\mathbb{R}^{3}$, and suppose $\gamma$ is $\mathcal{C}^{2}$ on $[0, L]$, with $\gamma^{\prime}(t) \neq 0, \forall t \in$ $[0, L]$. On a regular part of the curve (i.e. $\gamma(t), 0<t<L)$, the normal cone is simply $\gamma^{\prime}(t)^{\perp} \cap S^{2}$ (note that for a segment, the normal bundle is thus a cylinder). For the singular part (i.e. the two endpoints), we denote $S_{v}^{+}:=\{u \in$ $\left.S^{2},\langle u, v\rangle \geq 0\right\}$. One can easily show that the normal cone at $\gamma(0)$ and $\gamma(1)$ are 
$\{\gamma(0)\} \times S_{-\gamma^{\prime}(0)}^{+}$and $\{\gamma(1)\} \times S_{\gamma^{\prime}(1)}^{+}$respectively. These are two half spheres with a coherent orientation with respect to the normal bundle (independent of the parametrization).

Canonical orientation of $\mathcal{N}_{X} \cdot \partial X_{\varepsilon}$ being a closed hypersurface, it is canonically oriented and this induces a canonical orientation on the unit normal bundle of $X$ as follows : let $\left(e_{1}, \ldots, e_{d}\right)$ be an orthonormal basis of $\mathbb{R}^{d}$, and let $\left(\tau_{1}(x, n), \ldots, \tau_{d-1}(x, n)\right)$ an orthonormal basis of $T_{(x, n)} \mathcal{N}_{X}$ such that

$$
\left\langle\left(\pi_{p}+\varepsilon \pi_{n}\right)\left(\tau_{1}(x, n)\right) \wedge \cdots \wedge\left(\pi_{p}+\varepsilon \pi_{n}\right)\left(\tau_{d-1}(x, n)\right) \wedge n, e_{1} \wedge \cdots \wedge e_{d}\right\rangle>0,
$$

where $\pi_{p}$ denotes the projection on the spatial space, and $\pi_{n}$ the projection on the normal space : $\pi_{p}:(x, n) \in \mathbb{R}^{d} \times \mathbb{R}^{d} \mapsto x, \pi_{n}:(x, n) \in \mathbb{R}^{d} \times \mathbb{R}^{d} \mapsto n$. Then $\tau_{\mathcal{N}_{X}}(x, n):=\tau_{1}(x, n) \wedge \cdots \wedge \tau_{d-1}(x, n)$ is a simple $(d-1)$-vectorfield orienting $\mathcal{N}_{X}$. Expression (2) is independent of $0<\varepsilon<$ Reach $X$. Besides, the orientation of the normal bundle of $X$ does not depend on any orientation of $X$.

Definition 4 (Normal cycle). The normal cycle of a positive reach set $X \subset$ $\mathbb{R}^{d}$ is the $(d-1)$-current associated with $\mathcal{N}_{X}$ with its canonical orientation. For any differential form $\omega \in \Omega_{0}^{d-1}\left(\mathbb{R}^{d} \times \mathbb{R}^{d}\right)$, one has :

$$
N(X)(\omega):=\left[\mathcal{N}_{X}\right](\omega)=\int_{\mathcal{N}_{X}} \omega_{(x, n)}\left(\tau_{\mathcal{N}_{X}}(x, n)\right) d \mathcal{H}^{d-1}(x, n)
$$

Normal cycles for unions of sets with positive reach The theory of normal cycles can be extended to the case of finite unions of sets with positive reach, as done in [16. This allows to define normal cycles for a very large class of subsets. In particular it allows to define normal cycles for unions of segments, which will be used as our discrete models for curves. This extension can be stated as follows :

Theorem 1 (Additive property). There is a unique extension of normal cycles to finite unions of sets with positive reach such that the following property holds for any two such sets $C, S$ :

$$
N(C \cup S):=N(C)+N(S)-N(C \cap S)
$$

In fact this additive property holds for sets with positive reach, and is used recursively as definition in the case of unions of such sets. It can be shown that this definition is fully coherent.

\section{Metrics on Normal Cycles}

For our numerical purpose, we need a computable expression for the dissimilarity between shapes. In the very same spirit of [974], we will use a dual kernel metric on normal cycles as dissimilarity measure. This can be done by considering a kernel $K:\left(\mathbb{R}^{d} \times S^{d-1}\right)^{2} \rightarrow \mathcal{B}\left(\Lambda^{d-1}\left(\mathbb{R}^{d} \times \mathbb{R}^{d}\right), \mathbb{R}\right)$ of the form

$$
K((x, u),(y, v))(\alpha, \beta):=k_{p}(x, y) k_{n}(u, v)\langle\alpha, \beta\rangle
$$


where $x, y \in \mathbb{R}^{d}, u, v \in S^{d-1}, \alpha, \beta \in \Lambda^{d-1}\left(\mathbb{R}^{d} \times \mathbb{R}^{d}\right)$, and:

- $k_{p}(x, y)$ is a positive definite kernel on $\mathbb{R}^{d}$. In our experiments we used $k_{p}(x, y)=\exp \left(\frac{-|x-y|^{2}}{\sigma_{W}^{2}}\right)$, where $\sigma_{W}$ is a scale parameter.

- $k_{n}(u, v)$ is a positive definite kernel on $S^{d-1}$. We used the reproducing kernel of a Sobolev space $H^{s}\left(S^{d-1}\right)$ of order $s$. This kernel is in fact a scalar function of the distance between two vectors of $S^{d-1}$. Therefore it is rotation invariant and can be expanded in spherical harmonics, which will be useful for the numerical aspect.

- $\langle\cdot, \cdot\rangle$ is a scalar product between $d-1$-vectors in $\mathbb{R}^{d} \times \mathbb{R}^{d}$. We used a modification of the canonical scalar product between $d-1$-vectors that takes into account the parameter $\sigma_{W}$ to ensure invariance of the kernel metric when a rescaling is applied jointly to the data coordinates and $\sigma_{W}$. It is defined by

$$
\left\langle u_{1} \wedge \cdots \wedge u_{d-1}, v_{1} \wedge \cdots \wedge v_{d-1}\right\rangle:=\operatorname{det}\left(\left(\left\langle u_{i}, v_{j}\right\rangle_{\sigma_{V}}\right)_{1 \leq i, j \leq d-1}\right)
$$

where $\langle u, v\rangle_{\sigma_{V}}:=\frac{1}{\sigma_{V}^{2}}\left\langle\pi_{p}(u), \pi_{p}(v)\right\rangle_{\mathbb{R}^{d}}+\left\langle\pi_{n}(u), \pi_{n}(v)\right\rangle_{\mathbb{R}^{d}}$.

This kernel $K$ defines a Reproducing Kernel Hilbert Space (RKHS) $W$ of $(d-1)$-differential forms in $\mathbb{R}^{d} \times S^{d-1}$ and $\iota: W \hookrightarrow \Omega_{0}^{d-1}\left(\mathbb{R}^{d} \times S^{d-1}\right)$ is a continuous injection under some regularity conditions on the kernel (see [9], prop 18 , basically we require the continuity of the kernel, bounded, and vanishing at infinity ). $W^{\prime}$ has also a structure of Hilbert space, and $\Omega_{0}^{d-1}\left(\mathbb{R}^{d} \times S^{d-1}\right)^{\prime} \subset W^{\prime}$.

The key point of the model is the formula for the scalar product between two normal cycles in the dual space $W^{\prime}$ :

$$
\langle N(C), N(S)\rangle_{W^{\prime}}=\int_{\mathcal{N}_{C}} \int_{\mathcal{N}_{S}} k_{p}(x, y) k_{n}(u, v)\left\langle\tau_{\mathcal{N}_{S}}(x, u), \tau_{\mathcal{N}_{C}}(y, v)\right\rangle d \mathcal{H}^{d-1}(x, u) d \mathcal{H}^{d-1}(y, v)
$$

The dissimilarity between two shapes $S$ and $C$ is then defined as

$$
\|N(S)-N(C)\|_{W^{\prime}}^{2}=\langle N(S), N(S)\rangle_{W^{\prime}}-2\langle N(S), N(C)\rangle_{W^{\prime}}+\langle N(C), N(C)\rangle_{W^{\prime}}
$$

\section{Computational Framework}

The aim of this section is to derive the expression of the kernel metric on normal cycles (5) for unions of segments in $\mathbb{R}^{3}$, which we will use as approximations of real curves.

\subsection{Decomposition of the Normal Cycle for Unions of Segments}

Let $a, b \in \mathbb{R}^{3}$ and $C=[a, b]$ be the segment with extremities $a$ and $b$. We denote $\tilde{C}=C \backslash\{a, b\}$. As noticed in example 2 the normal bundle of $C$ is composed of two parts, a cylindrical part and a spherical part. More precisely, $\mathcal{N}_{C}=\mathcal{N}_{C}^{c y l} \cup \mathcal{N}_{C}^{s p h}$ with $\mathcal{N}_{C}^{c y l}:=\tilde{C} \times\left((b-a)^{\perp} \cap S^{2}\right)$ and $\mathcal{N}_{C}^{s p h}:=\left(\{a\} \times S_{a-b}^{+}\right) \cup$ 
$\left(\{b\} \times S_{b-a}^{+}\right)$. The normal cycle $N(C)$ thus satisfies $N(C)=N(C)^{c y l}+N(C)^{s p h}$ with $N(C)^{c y l}:=\left[\mathcal{N}_{C}^{c y l}\right]$ and $N(C)^{s p h}:=\left[\mathcal{N}_{C}^{s p h}\right]$.

In order to get a nice decomposition in the case of unions of segments, it is convenient to define the normal cycle associated to the "open" segment $\tilde{C}$ as: $N(\tilde{C}):=N(C)-N(\{a\})-N(\{b\})$. This definition is made on purpose to allow to extend the additive property (4) to such open segments. Since the normal bundles of $\{a\}$ and $\{b\}$ are entire spheres, we see that $N(\tilde{C})$ expresses also as a sum of a cylindrical part and a spherical part: $N(\tilde{C})=N(C)^{c y l}+N(\tilde{C})^{s p h}$ with $N(\tilde{C})^{s p h}:=-\left[\{a\} \times S_{b-a}^{+}\right]-\left[\{b\} \times S_{a-b}^{+}\right]$.

Now let $C=C_{1} \cup \cdots \cup C_{n}$ be a union of $n$ segments in $\mathbb{R}^{3}$. We can consider without loss of generality that the intersection of two segments $C_{i} \cap C_{j}$ is either empty or composed of a single point. If we denote $\left\{v_{1}, \ldots, v_{N}\right\}$ the set of end points of all segments $C_{i}, 1 \leq i \leq n$, we can rewrite $C$ as the disjoint union of the $\tilde{C}_{i}, 1 \leq i \leq n$, and the $\left\{v_{j}\right\}, 1 \leq j \leq N$. The additive property (4) then becomes straightforward and we get

$$
N\left(C_{1} \cup \cdots \cup C_{n}\right)=\sum_{i=1}^{n} N\left(\tilde{C}_{i}\right)+\sum_{j=1}^{N} N\left(\left\{v_{j}\right\}\right)
$$

which we can further decompose into cylindrical and spherical parts as follows :

$$
N\left(C_{1} \cup \cdots \cup C_{n}\right)=\left(\sum_{i=1}^{n} N\left(C_{i}\right)^{c y l}\right)+\left(\sum_{i=1}^{n} N\left(\tilde{C}_{i}\right)^{s p h}+\sum_{j=1}^{N} N\left(\left\{v_{i}\right\}\right)\right)
$$

\subsection{Computation of the Kernel Metric for Unions of Segments}

Let $C=C_{1} \cup \cdots \cup C_{n_{C}}, S=S_{1} \cup \cdots \cup S_{n_{S}}$ be two unions of segments. The calculation of the expression of (5) in this case is simplified by the following property:

Theorem 2. The cylindrical part and the spherical part are orthogonal with respect to the kernel metric presented in Sect. Q2.

This means we only need to compute scalar products between spherical parts, and scalar products between cylindrical parts. We do not give full details of this computations here and only sketch the main arguments. We denote $x_{1}, \ldots, x_{N_{C}}$ (resp. $y_{1}, \ldots, y_{N_{S}}$ ) the vertices of $C$ (resp. of $S$ ) and $f_{i}=x_{f_{i}^{2}}-x_{f_{i}^{1}}, 1 \leq i \leq n_{C}$ (resp. $g_{j}=y_{g_{j}^{2}}-y_{g_{j}^{1}}, 1 \leq j \leq n_{S}$ ) the edges of $C$ (resp. $S$ ). For an edge $f_{i}, x_{f_{i}^{1}}$ and $x_{f_{i}^{2}}$ are its two vertices. Moreover, we define $c_{i}=\frac{1}{2}\left(x_{f_{i}^{1}}+x_{f_{i}^{2}}\right), d_{j}=\frac{1}{2}\left(y_{g_{j}^{1}}+y_{g_{j}^{2}}\right)$ and, $\theta_{i j}=\arccos \left(\left\langle\frac{f_{i}}{\left|f_{i}\right|}, \frac{g_{j}}{\left|g_{j}\right|}\right\rangle\right)$.

For the cylindrical part, in (5), the point kernel integrated over the segment is approximated by its value at the center, with a coefficient taking into account the length of the edge. For the normal part, we use an expansion in spherical harmonics of $k_{n}$ (which is valid since $k_{n}$ is rotation invariant), and pre integrate the kernel on the normal parts. The result is a quantity depending on the angle 
between the edges. For the spherical part, the evaluation of the point kernel is immediate and we use the same argument for the normal kernel. Finally we get

$$
\left\langle N(C)^{c y l}, N(S)^{c y l}\right\rangle_{W^{\prime}} \simeq \frac{1}{\sigma_{W}^{2}} \sum_{i=1}^{n_{C}} \sum_{j=1}^{n_{S}} k_{p}\left(c_{i}, d_{j}\right)\left\langle f_{i}, g_{j}\right\rangle \sum_{m \geq 0} a_{m} \cos \left(m \theta_{i j}\right)
$$

and

$$
\begin{aligned}
\left\langle N(C)^{s p h}, N(S)^{s p h}\right\rangle_{W^{\prime}} & =\sum_{k=1}^{N_{C}} \sum_{l=1}^{N_{S}} k_{p}\left(x_{k}, y_{l}\right)\left(1-\frac{n_{x_{k}}+n_{y_{l}}}{2}\right) \beta \\
& +\sum_{i=1}^{n_{C}} \sum_{j=1}^{n_{S}} \sum_{a, b=1}^{2}\left(b_{0}+(-1)^{a+b} \sum_{m \geq 0} b_{m} \cos \left(m \theta_{i j}\right)\right) k_{p}\left(x_{f_{i}^{a}}, y_{g_{j}^{b}}\right)
\end{aligned}
$$

where $n_{x_{k}}$ (resp. $n_{y_{l}}$ ) is the number of edges adjacent to the vertex $x_{k}$ (resp. $y_{l}$ ).

The constant $\beta$ and the $a_{m}$ and $b_{m}$ coefficients have explicit expansions in spherical harmonics, and are pre-computationable. Even though they are not detailed here, we just precise the fact that they vanish for $m$ even. This ensures that if we invert the orientation of the edges (i.e. if we invert $x_{f_{i}^{1}}$ and $x_{f_{i}^{2}}$ ), the scalar product remains unchanged. We retrieve here the fact that normal cycles are unoriented objects. With these two scalar products, we have all we need to implement an algorithm which computes dissimilarity between two discrete curves. This is the first step to have a matching algorithm.

\section{Curve Matching via Normal Cycles}

Given two curves $C, S$ in $\mathbb{R}^{3}$, we define the curve matching problem as the minimization of a functional over a given set of deformations $G$. This functional takes the form

$$
\varphi_{0}=\arg \min _{\varphi \in G} E(\varphi)+A(N(\varphi(C)))
$$

where $A(N(\varphi(C)))=\|N(\varphi(C))-N(S)\|_{W^{\prime}}^{2}$ is the data attachment term evaluating the dissimilarity between the deformed curve $\varphi(C)$ and the target $S$, and $E(\varphi)$ is an energy which ensures regularity of the mapping. In our experiments we chose to use the Large Deformation Diffeomorphic Metric Mapping (LDDMM) framework for defining the space $G$ of deformations and the energy $E$, but of course other frameworks for non-rigid registration could be used, such as for example Thin Plate Splines ([3]).

In a discrete setting, curves are given as unions of segments. As a first approximation, we replace $\varphi(C)$ by the union of segments corresponding to moving only the vertices of $C$ (we do not consider the deformation of all the curve). Note that it is possible to define a geometric action of a diffeomorphism $\varphi$ of $\mathbb{R}^{d}$ on normal cycles, by considering the diffeomorphism induced by $\varphi$ on $\mathbb{R}^{d} \times S^{d-1}$ and the standard push forward action on currents. However we do not use this action in this work. 


\subsection{Gradient of the Data Attachment Term Associated with Normal Cycle}

As explained previously, we suppose that the deformation acts only on the vertices. Thus, we can consider that $A$ is a function of the current position of the vertices of $C: A\left(\varphi\left(x_{k}\right)_{1 \leq k \leq N}\right)$. This function can be computed explicitely using (6) and the expressions for the scalar products (8), (9). Then a numerical implementation of the minimization of 10 requires the computation of $\left.\nabla A\left(\left(x_{k}\right)_{1 \leq k \leq N}\right)\right)$, which takes an explicit form by deriving these expressions. We do not detail this calculation here.

\subsection{Large Deformation Diffeomorphic Metric Mapping (LDDMM)}

The classical LDDMM framework as explained for example in [2] is a variational problem :

$$
\left\{\begin{aligned}
\min _{v \in L^{2}([0,1], V)} J(v) & :=\gamma\left(\int_{0}^{1}\left\|v_{t}\right\|_{V}^{2} d t\right)+A\left(\varphi_{1}(C)\right) \\
\frac{\partial \varphi_{t}}{\partial t} & =v_{t} \circ \varphi_{t}
\end{aligned}\right.
$$

where $V$, the space of deformation is chosen to be a RKHS with kernel $K_{V}$. In this article, $K_{V}$ will be a Cauchy kernel, with width $\sigma_{V}: K_{V}(x, y)=\frac{1}{1+\frac{|x-y|^{2}}{\sigma_{V}^{2}}}$. We can show in the same spirit as [9] (Chap. 1, Prop. 9 and Chap. 5, Prop 34) that if $V \hookrightarrow \mathcal{C}_{0}^{3}\left(\mathbb{R}^{d}, \mathbb{R}^{d}\right)$, then there exists a minimum for problem (11).

As explained previously, we only consider at the discrete level the deformation of $N$ points $\left(x_{i}\right)_{1 \leq i \leq N}$ in $\mathbb{R}^{3}$ (the vertices of discrete curves). As shown in [9], if we denote by $q_{i}(t)=\varphi_{t}\left(x_{i}\right)$ the points trajectories, the optimal vector field minimizing (11) is necessarily of the form

$$
v_{t}=\sum_{i=1}^{N} K_{V}\left(\cdot, q_{i}(t)\right) p_{i}(t)
$$

where the $p_{i}(t) \in \mathbb{R}^{3}$ are called momentum vectors. Further, it was shown in 11] that the problem can be written in Hamiltonian form and that $q_{i}(t)$ and $p_{i}(t)$ must satisfy geodesic equations which write

$$
\left\{\begin{array}{l}
\dot{q}_{i}(t)=\sum_{j=1}^{N} K_{V}\left(q_{i}(t), q_{j}(t)\right) p_{j}(t) \\
\dot{p}_{i}(t)=-\left(d_{q_{i}(t)} v_{t}\right)^{*} p_{i}(t)
\end{array}\right.
$$

Initial positions $q_{i}(0)$ being fixed, we can consider the mapping $\varphi$ and further functional $J$ as function of the $p_{i}(0)$ only. This property allows to derive an algorithm which optimizes only on these initial momentum vectors, which reduces significantly the dimensionality of the problem. This algorithm is called geodesic shooting ([11]1]). 


\section{Application to 3D Curve Matching}

\subsection{Algorithm}

We use the shooting algorithm and optimize the functional depending on $p_{0}$ with a quasi Newton Broyden Fletcher Goldfarb Shanno algorithm with limited memory (L-BFGS) [10. The step in the descent direction is fixed by a Wolfe line search. For the numerical integrations, a Runge-Kutta $(4,5)$ scheme is used (function ode45 in Matlab). For the normal cycles, the point kernel $k_{p}$ is a Gaussian kernel, with width $\sigma_{W}$, and the normal kernel $k_{n}$ is a Sobolev kernel, associated with the operator $L=(I-\Delta)^{2}$. We used a spherical harmonics expansion of this kernel truncated at order 5 for the numerical purpose. All the numerical computations have been done on a laptop using Matlab.

\subsection{Numerical Results on Synthetic Data}

In this section, we show some of our results on synthetic data and compare them with the varifolds method and currents method. The point kernel chosen for the varifolds is a Gaussian kernel, with the same width $\sigma_{W}$ as for normal cycles. The kernel associated with the Grassmanian is chosen linear (see [4]), so that no parameter is involved as for the normal kernel with normal cycles. Lastly, a Gaussian kernel is used as well for currents, again with width $\sigma_{W}$. The trade-off parameter $\gamma$ is fixed for normal cycles : $\gamma=0.1$ (since a factor $\frac{1}{\sigma_{W V}^{2}}$ appears in the cylindrical scalar product, the balance between the two terms is also modified with the kernel width $\sigma_{W}$ ). For currents and varifolds, we set the parameter $\gamma$ to be consistent with the metric on normal cycles : we set $\gamma=0.1 \times \sigma_{W}^{2}$.

Registrations of branching curves (Fig. 1) The first example of registration is two 3D curves with branching. These curves were chosen because the distance between them is large compared to their typical sizes, the curves have some high local curvature and the size of the corresponding branches implies high local deformations. Besides, we would like to see the behaviour of normal cycles with respect to connecting points.

The two curves are enclosed in a cubic box of size one. Both curves have 150 vertices. In Fig. 1 we show two views of a matching using normal cycles, varifolds and currents. The kernel $K_{V}$ associated to the deformation space is chosen to be a Cauchy kernel, with width $\sigma_{V}=0.2$. Computation time for registration with currents and varifolds were 37 and 120 seconds respectively. Computations with normal cycles are more expensive and took 580 seconds.

As we can see in Fig. 1 w we get a nearly perfect registration with normal cycles. The connecting points of the two curves are well matched, as well as the end points. This is not the case with varifolds and currents, which give less accurate matchings on this example. 


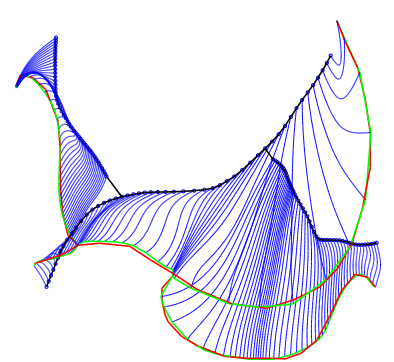

(a) Normal cycles, view 1



(c) Varifolds, view 1

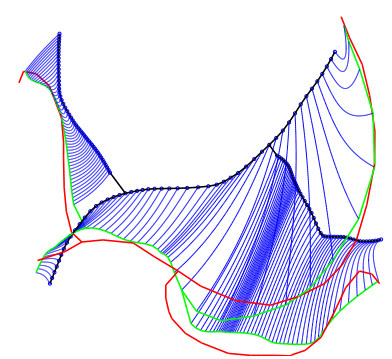

(e) Currents, view 1

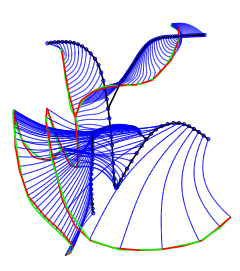

(b) Normal cycles, view 2

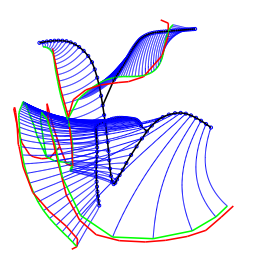

(d) Varifolds, view 2

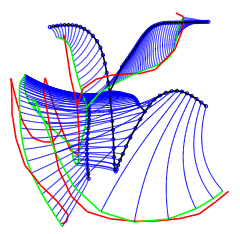

(f) Currents, view 2

Fig. 1: Registration of two 3D curves with different data attachment terms. Initial curve is in black, target curve in red, and deformed curve in green. Trajectories of vertices along the flow are displayed in blue. Parameters are $\sigma_{V}=0.2$ and $\sigma_{W}=0.3$ 
Registration of fishes contours (Fig. 2) Here a registration between two fishes contours is performed (see [14] for the original data). Even if they are 2D objects, we consider them as 3D objects with no $z$ variation. In this example, fishes have around 100 vertices. A first optimization of the momenta was performed with parameters $\sigma_{W}=0.75$ and $\sigma_{V}=0.2$. This can be seen as an initialization step to avoid local minima. Then minimization was done with $\sigma_{W}=0.2$ and $\sigma_{V}=0.2$. Computation time was 310 seconds for normal cycles and 60 seconds for varifolds. The main difficulty here is the trade off to find between the matching of the long tail of the stingray (in green in Fig. 2) and the high local curvature in the upper part of the fish in dark. The results in Fig. 2 show that a perfect matching with normal cycles can be achieved, even with $\sigma_{W}=0.2$ which is quite large compared to the local feature in the upper part of the fish. With varifolds, one can see that this local feature still remains in the green matched curve. To avoid this behaviour, one can decrease the size of $\sigma_{W}$, but it would lead to a bad matching of the tail.

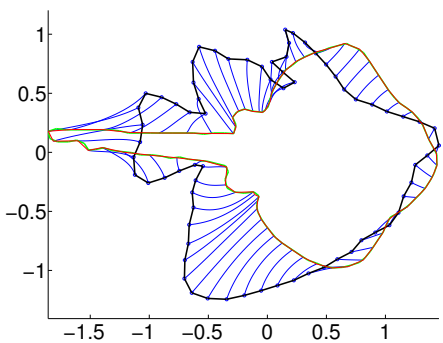

(a) Normal cycles

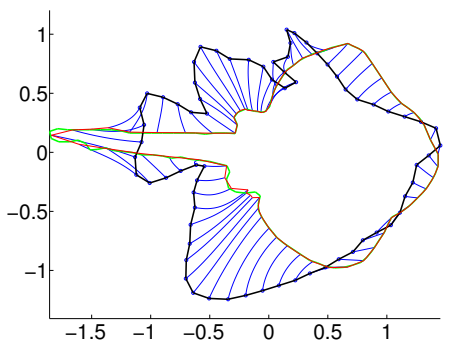

(b) Varifolds

Fig. 2: Registration of a dark fish to a red fish. In green the dark deformed fish matching the red one. We used normal cycles and varifolds with the same parameters $\sigma_{V}=0.2$ and $\sigma_{W}=0.2$. The registration with currents is worse than with varifolds.

\section{Perspectives}

In this article, we have presented the first application of normal cycles in the context of 3D curve registration. As for currents, a kernel metric is used to provide a closed form for the distance between two curves, and a numerical derivation is done for curves approximated by unions of segments. The first results on synthetic data are promising and suggest that the normal cycles metric improves matchings between connection points and regions with high curvature. Of course, wether such a property is desirable in real applications remains an open debate and highly depends on the type of data in use. Moreover, more exhaustive studies on synthetic and real data are necessary to validate the method. 
The next stage will be the registration using normal cycles for surfaces. This case is more intricate, at least numerically since the decomposition of normal bundle as seen in Sect. 3 is more complex. We also would like to investigate the link between varifolds and normal cycles, as we believe that varifolds can be seen in our context as a projection of normal cycles, by ignoring variation in $S^{d-1}$.

\section{References}

1. Arguillere, S., Trélat, E., Trouvé, A., Younes, L.: Shape deformation analysis from the optimal control viewpoint. arXiv:1401.0661 [math] (Jan 2014)

2. Beg, M.F., Miller, M.I., Trouvé, A., Younes, L.: Computing Large Deformation Metric Mappings via Geodesic Flows of Diffeomorphisms. International Journal of Computer Vision 61(2), 139-157 (Feb 2005)

3. Bookstein, F.L.: Principal Warps: Thin-Plate Splines and the Decomposition of Deformations. IEEE Transactions on Pattern Analysis and Machine Intelligence 11(6), 567-585 (1989)

4. Charon, N.: Analysis of geometric and functionnal shapes with extension of currents. Application to registration and atlas estimation. Ph.D. thesis, École Normale Supérieure de Cachan (2013)

5. Charon, N., Trouvé, A.: The varifold representation of nonoriented shapes for diffeomorphic registration. SIAM J. Imaging Sciences 6(4), 2547-2580 (2013)

6. Cohen-Steiner, D., Morvan, J.M.: Restricted Delaunay Triangulations And Normal Cycle. SoCG'03 (2003)

7. Durrleman, S.: Statistical models of currents for measuring the variability of anatomical curves, surfaces and their evolution. Ph.D. thesis, Université Nice Sophia Antipolis (2010)

8. Federer, H.: Curvature measures. Trans. Amer. Maths. Soc. 93 (1959)

9. Glaunès, J.: Transport par difféomorphismes de points, de mesures et de courants pour la comparaison de formes et l'anatomie numérique. Ph.D. thesis, Université Paris 13 (2005)

10. Liu, D.C., Nocedal, J.: On the limited memory BFGS method for large scale optimization. Mathematical Programming 45(1-3), 503-528 (Aug 1989)

11. Miller, M.I., Trouvé, A., Younes, L.: Geodesic Shooting for Computational Anatomy. Journal of Mathematical Imaging and Vision 24(2), 209-228 (Jan 2006)

12. Morvan, J.M.: generalized curvatures. Springer (2008)

13. Vaillant, M., Glaunès, J.: Surface Matching via Currents. In: Christensen, G.E., Sonka, M. (eds.) Information Processing in Medical Imaging, pp. 381-392. No. 3565 in Lecture Notes in Computer Science, Springer Berlin Heidelberg (2005)

14. US Dept of the Interior Fish and Wildlife Service: Fishes of the gulf of maine (1953), http://www.nefsc.noaa.gov/lineart/

15. Zähle, M.: Integral and current representation of Federer's curvature measure. Arch. Maths. 23, 557-567 (1986)

16. Zähle, M.: Curvatures and currents for unions of set with positive reach. Geometriae Dedicata 23, 155-171 (1987) 